\title{
NONPARAMETRIC MULTI-LEVEL CLUSTERING OF HUMAN EPILEPSY SEIZURES SUPPLEMENTARY MATERIALS
}

\author{
By Drausin Wulsin*, Shane Jensen ${ }^{\dagger}$, And Brian Litt ${ }^{*}$, \\ Depts. of Bioenginering ${ }^{*}$, Statistics ${ }^{\dagger}, \mathcal{E}$ Neurology ${ }^{\ddagger}$ \\ University of Pennsylvania
}

\section{CONTENTS}

1 Normal model with a conjugate prior . . . . . . . . . . . . 1

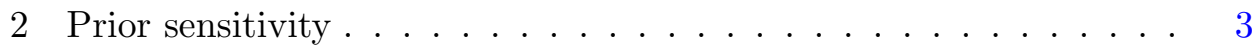

3 Algorithms for posterior implementation . . . . . . . . . . . 6

4 Simulation comparison to the NDP . . . . . . . . . . . 13

5 Schiff seizure features . . . . . . . . . . . . . . 15

6 Alternative sampling schemes . . . . . . . . . . . . . 16

7 The influence of individual patients . . . . . . . . . . . . . 18

8 Patient Seizure Images . . . . . . . . . . . . . . . . . . . 20

References . . . . . . . . . . . . . . . . . . 21

1. Normal model with a conjugate prior. Here we briefly describe the details of our Normal scaled inverse- $\chi^{2}$ conjugate prior for a univariate Normal and the resulting posterior. For more details, see (Gelman et al. 2004, pgs. 78-80).

Our base model is a multivariate Normal with mean $\boldsymbol{\mu}$ and diagonal covariance $\boldsymbol{\sigma}^{2}$, both in $\mathbf{R}^{d}$. The diagonal covariance implies that our $d$ dimensions are independent, so the likelihood of $\mathbf{x}$ is simply the product of the each dimension $i$ 's likelihood of $x_{i}$, and sampling $\boldsymbol{\mu}$ and $\boldsymbol{\sigma}^{2}$ from the posterior just involves sampling each dimension individually. For notational ease, we will thus assume that $d=1$ in what follows below, so $\mathbf{x}=x$ and $\left(\boldsymbol{\mu}, \boldsymbol{\sigma}^{2}\right)=\left(\mu, \sigma^{2}\right)$.

The Normal model likelihood is

$$
p\left(x \mid \mu, \sigma^{2}\right) \propto\left(\sigma^{2}\right)^{-1 / 2} \exp \left(-\frac{1}{2 \sigma^{2}}(x-\mu)^{2}\right)
$$

Given $n$ i.i.d. observations $x_{1}, \ldots, x_{n}$, their joint likelihood is

$$
p\left(x_{1}, \ldots, x_{n} \mid \mu, \sigma^{2}\right) \propto\left(\sigma^{2}\right)^{-n / 2} \exp \left(-\frac{1}{2 \sigma^{2}} \sum_{i=1}^{n}\left(x_{i}-\mu\right)^{2}\right)
$$


We assume that both the mean and variance of our Normal distribution are unknown, requiring a Normal scaled inverse- $\chi^{2}$ conjugate prior, which entails

$$
\begin{aligned}
\sigma^{2} & \sim \operatorname{Inv}-\chi^{2}\left(\nu_{0}, \sigma_{0}^{2}\right) \\
\mu \mid \sigma^{2} & \sim \mathcal{N}\left(\mu_{0}, \sigma^{2} / n_{0}\right)
\end{aligned}
$$

and has a joint prior density, denoted $\mathcal{N}-\operatorname{Inv}-\chi^{2}\left(n_{0}, \mu_{0}, \nu_{0}, \sigma_{0}^{2}\right)$, of

$$
p\left(\mu, \sigma^{2}\right) \propto\left(\sigma^{2}\right)^{-\left(\nu_{0}+1\right) / 2+1} \exp \left(-\frac{1}{2 \sigma^{2}}\left(\nu_{0} \sigma_{0}^{2}+n_{0}\left(\mu_{0}-\mu\right)^{2}\right)\right)
$$

where $n_{0}$ is the prior counts, $\mu_{0}$ is the prior location, $\nu_{0}$ is the prior degrees of freedom, and $\sigma_{0}^{2}$ is the prior scale.

The posterior density is the product of the data likelihood and the joint prior and has the form

$p\left(x_{1}, \ldots, x_{n} \mid \mu, \sigma^{2}\right) \propto\left(\sigma^{2}\right)^{-\left(\nu_{0}+n+1\right) / 2+1} \exp \left(-\frac{1}{2 \sigma^{2}}\left(\sum_{i=1}^{n}\left(x_{i}-\mu\right)^{2}+\nu_{0} \sigma_{0}^{2}+n_{0}\left(\mu_{0}-\mu\right)^{2}\right)\right)$

with posterior parameters $\bar{n}, \bar{\mu}, \bar{\nu}$, and $\bar{\sigma}^{2}$, which we can efficiently represent using the sufficient statistics (Sudderth 2006)

$$
\begin{aligned}
\bar{n} & =n_{0}+n \\
\bar{n} \bar{\mu} & =n_{0} \mu_{0}+\sum_{i=1}^{n} x_{i} \\
\bar{\nu} & =\nu_{0}+n \\
\bar{\nu} \bar{\sigma}^{2} & =\nu_{0} \sigma_{0}^{2}+n_{0} \mu_{0}^{2}+\sum_{i=1}^{n} x_{i}^{2}-\bar{n} \bar{\mu}^{2}
\end{aligned}
$$

These values can be cached for each base atom and easily updated when a new data point is added or removed. In practice, we only store the first three terms of (9) and subtract off the last term when we need to actually use the sufficient statistics (e.g., for sampling $\mu$ and $\sigma^{2}$ values from the posterior). In situations where full covariance is required, the setup is still quite similar, except the Cholesky decomposition of the analogous form of (9) is stored (see (Sudderth 2006) for more details).

We sample from the posterior in two steps, first drawing $\sigma^{2}$ from its marginal posterior distribution

$$
\sigma^{2} \sim \operatorname{Inv}-\chi^{2}\left(\bar{\nu}, \bar{\sigma}^{2}\right)
$$

imsart-aoas ver. 2011/12/06 file: mlchdpAOAS.supp_v05.tex date: January 3, 2016 
or (in practice)

$$
\begin{aligned}
\theta & \sim \operatorname{Inv}-\chi^{2}(\bar{\nu}) \\
\sigma^{2} & =\bar{\nu} \bar{\sigma}^{2} / \theta
\end{aligned}
$$

and then drawing $\mu$ from its conditional posterior distribution

$$
\mu \mid \sigma \sim \mathcal{N}\left(\bar{\mu}, \sigma^{2} / \bar{n}\right)
$$

Integrating over the $\boldsymbol{\mu}$ and $\boldsymbol{\sigma}^{2}$ parameters of the $\mathcal{N}$-Inv- $\chi^{2}$ distribution yields the posterior predictive distribution, here a Student- $t$ (Gelman et al. 2004, pg. 88), for a new data point $x^{+}$,

$$
p\left(x^{+} \mid x_{1}, \ldots, x_{n}, n_{0}, \mu_{0}, \nu_{0}, \sigma_{0}^{2}\right)=t_{\bar{\nu}}\left(x^{+} \mid \bar{\mu}, \frac{\bar{n}+1}{\bar{n}} \bar{\sigma}^{2}\right)
$$

2. Prior sensitivity. Throughout our paper, we have intentionally used vague priors since we have very little basis for anything stronger. We generally set the observation model prior for the base distribution from the data but have no such convenient method for the gamma priors on the $\alpha$ and $\gamma$ concentration parameters at each of the three model levels. We thus varied the gamma prior shape parameter between 1 and 100 for each of the six concentration parameters in turn (and keeping the other five fixed at the values given in our paper) to assess how sensitive the global model performance was to these priors. We used the average patient clustering performance (relative to the same gold-standard markings used in the paper) for our metric of global model performance. We ran 10 chains, taking 100 samples per chain at 20-iteration spacing after 500-iteration burn-in, for 18 different shape values for each of the 6 concentration parameters. For each MCMC sample and each of the eight patients, we calculate the $c$-statistic between the MLC-HDP model's seizure clustering and the physician gold-standard clustering of that patient as described in our paper. These $c$-statistics are averaged across patients and MCMC samples to yield a single value for the given gamma prior shape value for the particular concentration parameter.

The right side of Figure 5 shows these results for each of the six concentration parameters. We notice that varying the prior shape parameter over much larger values than those normally used has very little effect on the global clustering performance. We also ran a permutation test on goldstandard physician seizure clusterings to obtain a null distribution for the mean $c$-statistic across the patients. Over 100,000 permutations, we found the $95 \%$ confidence interval of the mean $c$-statistic null-distribution to be $[0.48,0.64]$, well below the values around 0.8 yielded by the MLC-HDP.

imsart-aoas ver. 2011/12/06 file: mlchdpAOAS.supp_v05.tex date: January 3, 2016 

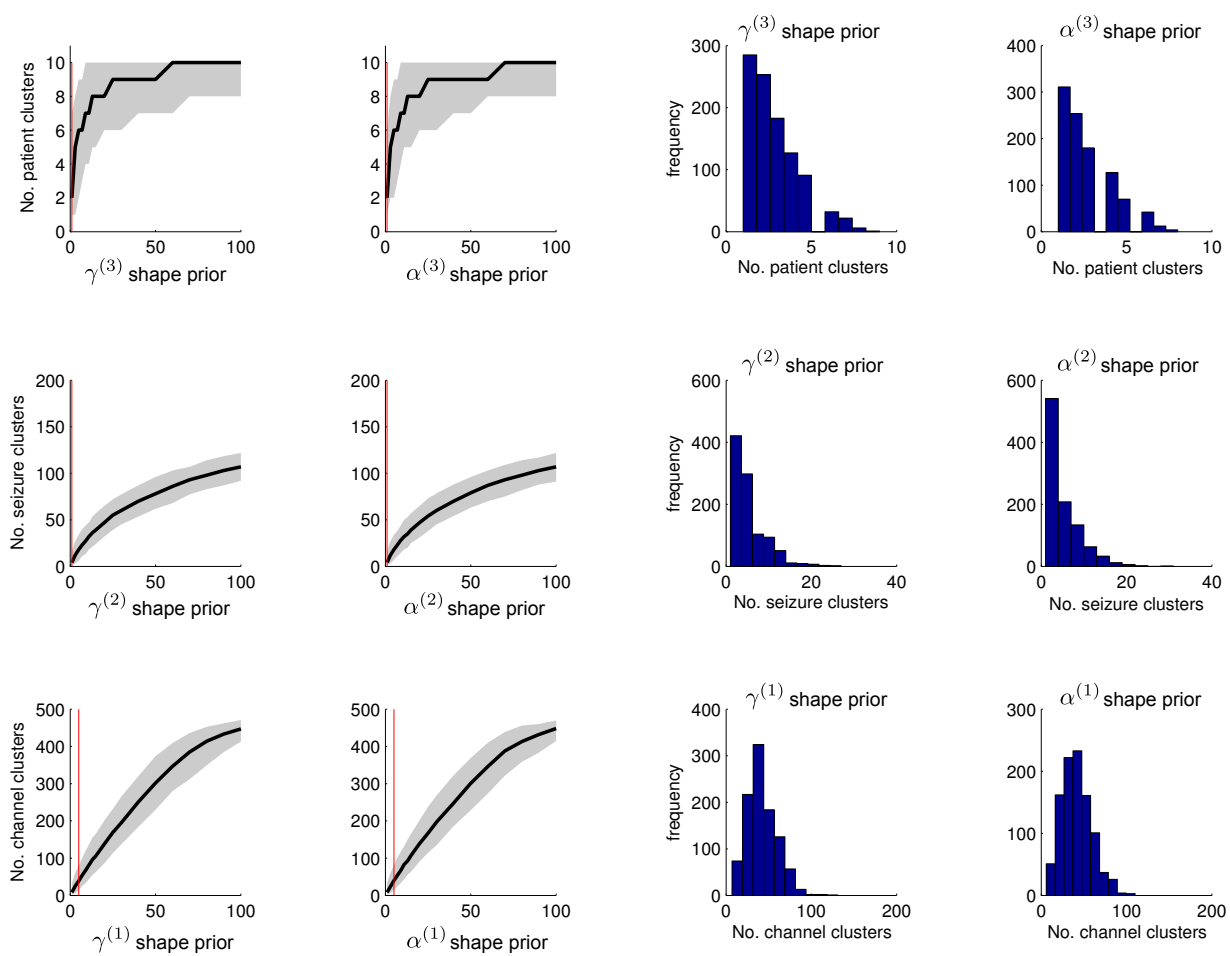

FIG 1. (first two columns) The number of clusters produced at each level of the hierarchy in prior simulations over a range of Gamma prior shape values for the two concentration paramters at each level. The black lines denote the distribution median and the gray area the $95 \%$ confidence region. The red line denotes the actual Gamma prior shape value used for the seizure experiments in the main paper. (last two columns) Histograms of the number of clusters produced at each level of the hierarchy in prior simulations for the Gamma prior shape values used in the seizure experiments of the main paper (i.e., the cross section distribution at the red lines in the first two columns of the figure). 

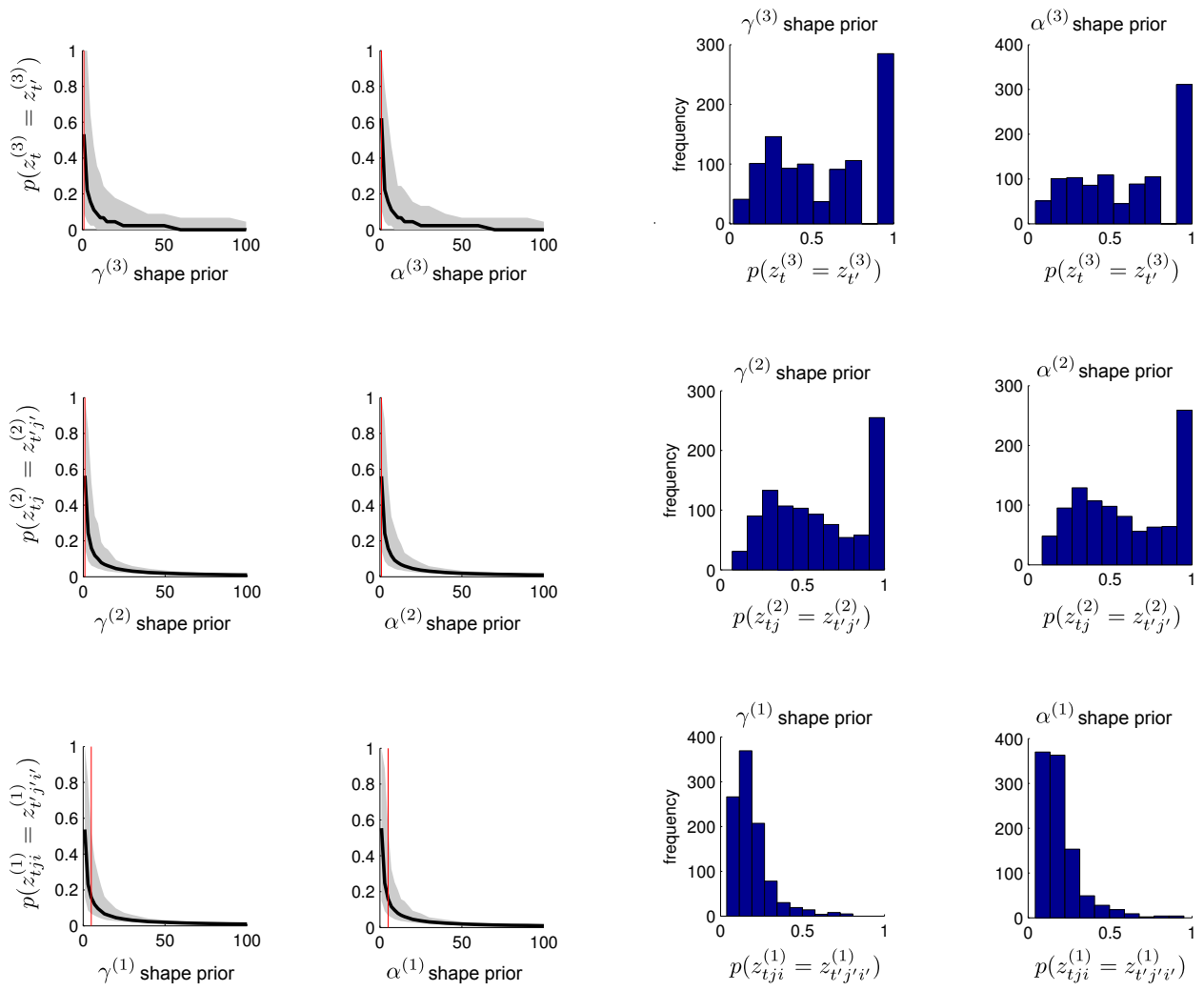

FIG 2. (first two columns) The probability of any two channels $\left(t j i \neq t^{\prime} j^{\prime} i^{\prime}\right)$, seizures $\left(t j \neq t^{\prime} j^{\prime}\right)$, or patients $\left(t^{\prime} \neq t^{\prime}\right)$ being clustered together over a range of Gamma prior shape values for the two concentration parameters. The black lines denote the distribution median and the gray area the $95 \%$ confidence region. The red line denotes the actual Gamma prior shape value used for the seizure experiments in the main paper. (last two columns) Histograms of the probability of two channels, seizures, or patients being clustered together produced at each level of the hierarchy in prior simulations for the Gamma prior shape values used in the seizure experiments of the main paper (i.e., the cross section distribution at the red lines in the first two columns of the figure). 
To fully understand how the prior distributions on our $\gamma^{v}$ and $\alpha^{v}$ hyperparameters affects our clustering characteristics, we simulated clusterings at each level from the priors over the same range of Gamma prior shape parameters used above. $\mathrm{T}$ his simulation used the same Rao-Blackwellized sampler described in the paper. This prior simulation scheme results in the clusterings at each level being independent. Thus, changing a parameter on one level has no influence on the prior clustering of a different level. We clustered the same number of channels, seizures, and patients at each level as existed in our original seizure dataset $(21,476,193$, and 10, respectively). We gathered 1000 samples for each of the 18 Gamma prior shape values for each of six concentration parameters.

Figure 1 shows the number of clusters yielded at each level of the model. As expected, the number of clusters increases as the Gamma prior shape parameter increases. The prior distribution in the number of clusters at each level for the prior values used in our seizure experiments is similarly reasonable.

Figure 2 shows the probability of clustering two different channels, seizures, and patients at each of their respective level. As the number of clusters increases with increasing Gamma prior shape, the probability of any two items being clustered together goes down, as we would expect. These distributions show broad support over the $[0,1]$ domain, especially for the seizure and patient clusters. The distributions in the number of clusters produced at each level and the probability of two channels, seizures, or patients being clustered together are broad but reasonable for our iEEG dataset.

In summary, though the prior distributions do change depending on these wide range of hyperparameter values, we see that our posterior inference is not sensitive to these differing hyperparameter values.

3. Algorithms for posterior implementation. In the algorithms below, we use the nomenclature of our epilepsy application - channels, seizures, and patients - to denote the organization of the data at each level of the model, though of course the model itself is agnostic to the nomenclature of this organization.

The function $\Upsilon(\mathbf{x})$ calculates the sufficient statistics of observation $\mathbf{x}$ for the model posterior. The function $f_{0}(\mathbf{x})$ denotes the prior predictive distribution. Similar to Matlab synatax, we use a semicolon to denote vertical matrix concatenation. We denote sampling a scalar $z$ from a multinomial distribution with vector weights $\mathbf{p}$ as $z \sim \operatorname{Multi}(\mathbf{p})$, where the weights $\mathbf{p}$ need not be normalized. A $*$ in place of an index indicates that all indices are selected, yielding a vector. Recall that a dot $(\cdot)$ in a subscript denotes

imsart-aoas ver. 2011/12/06 file: mlchdpAOAS.supp_v05.tex date: January 3, 2016 
summing over the dimension that the subscript indexes.

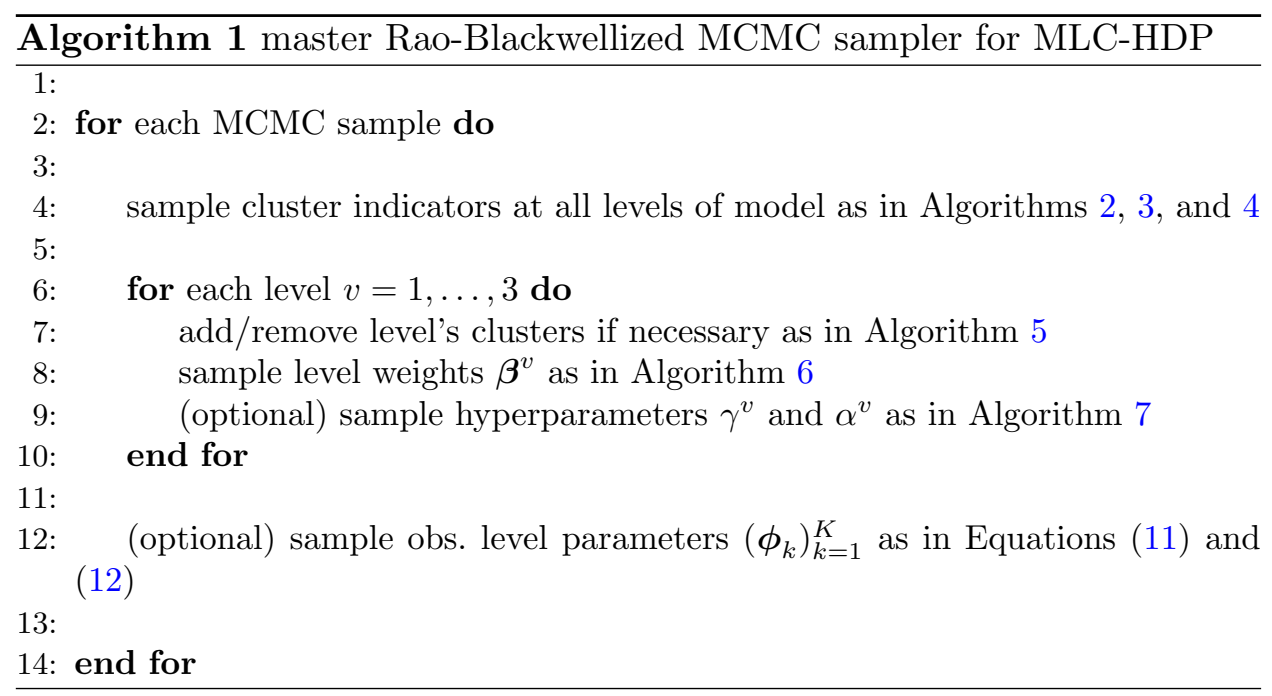




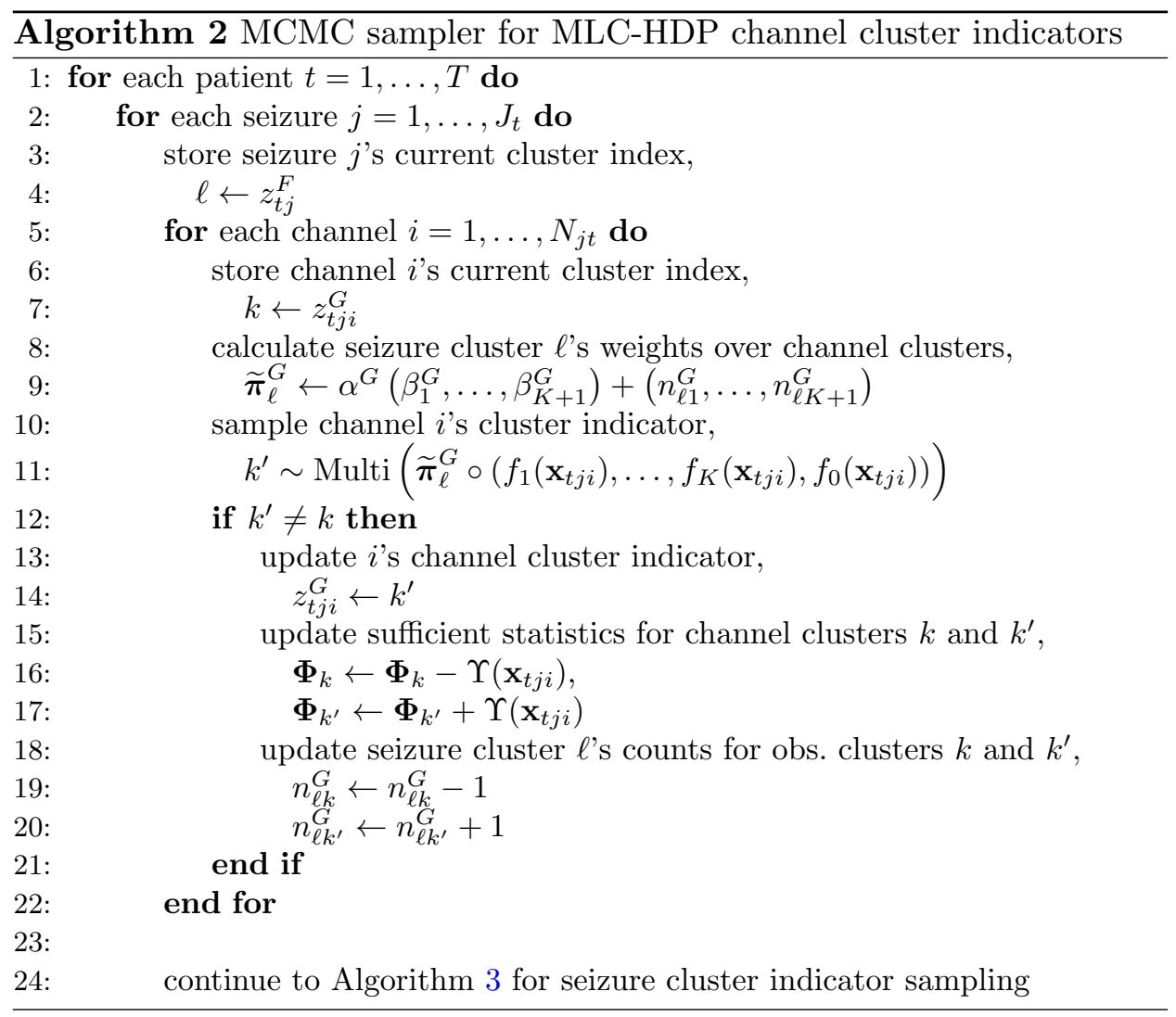




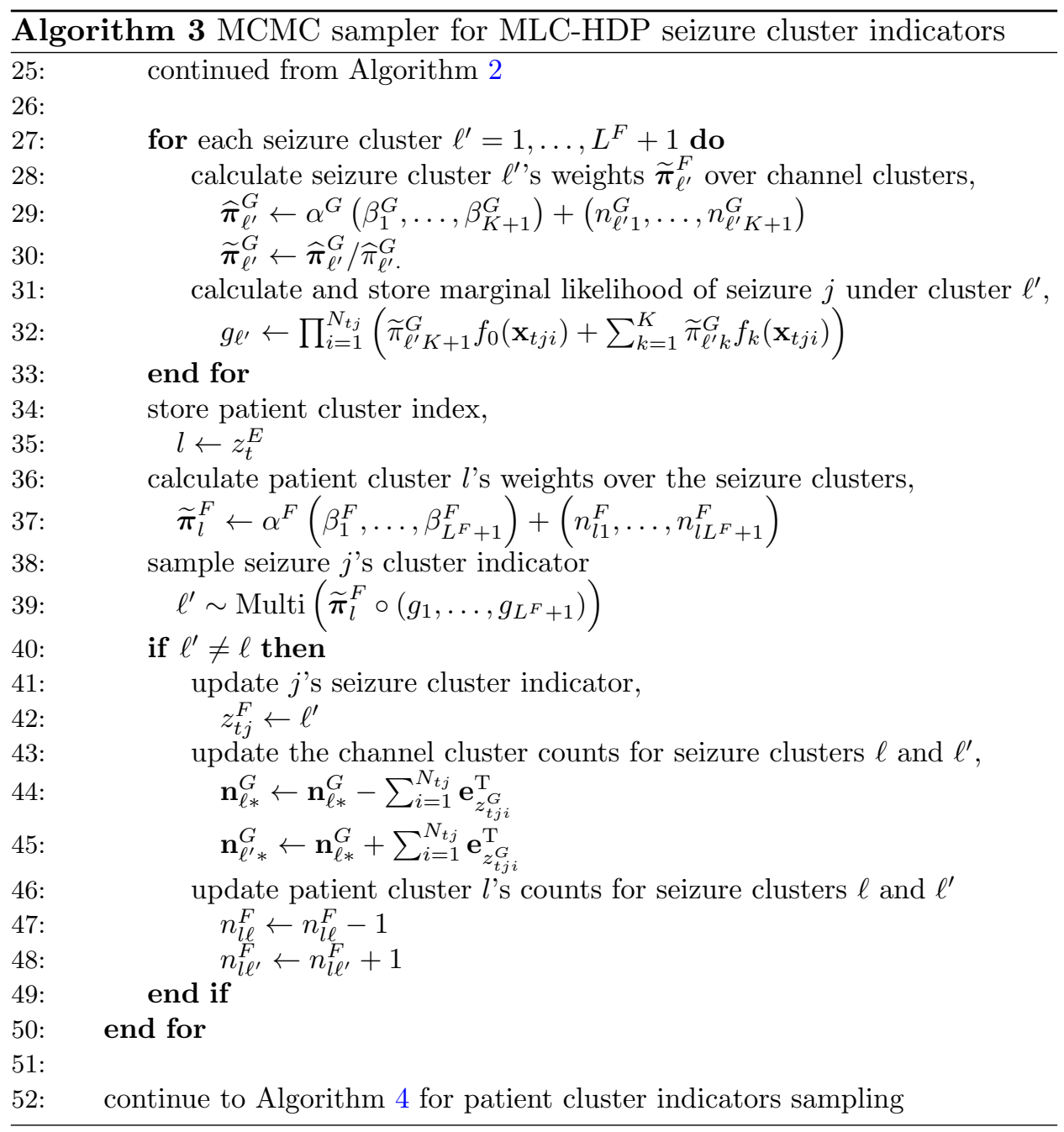




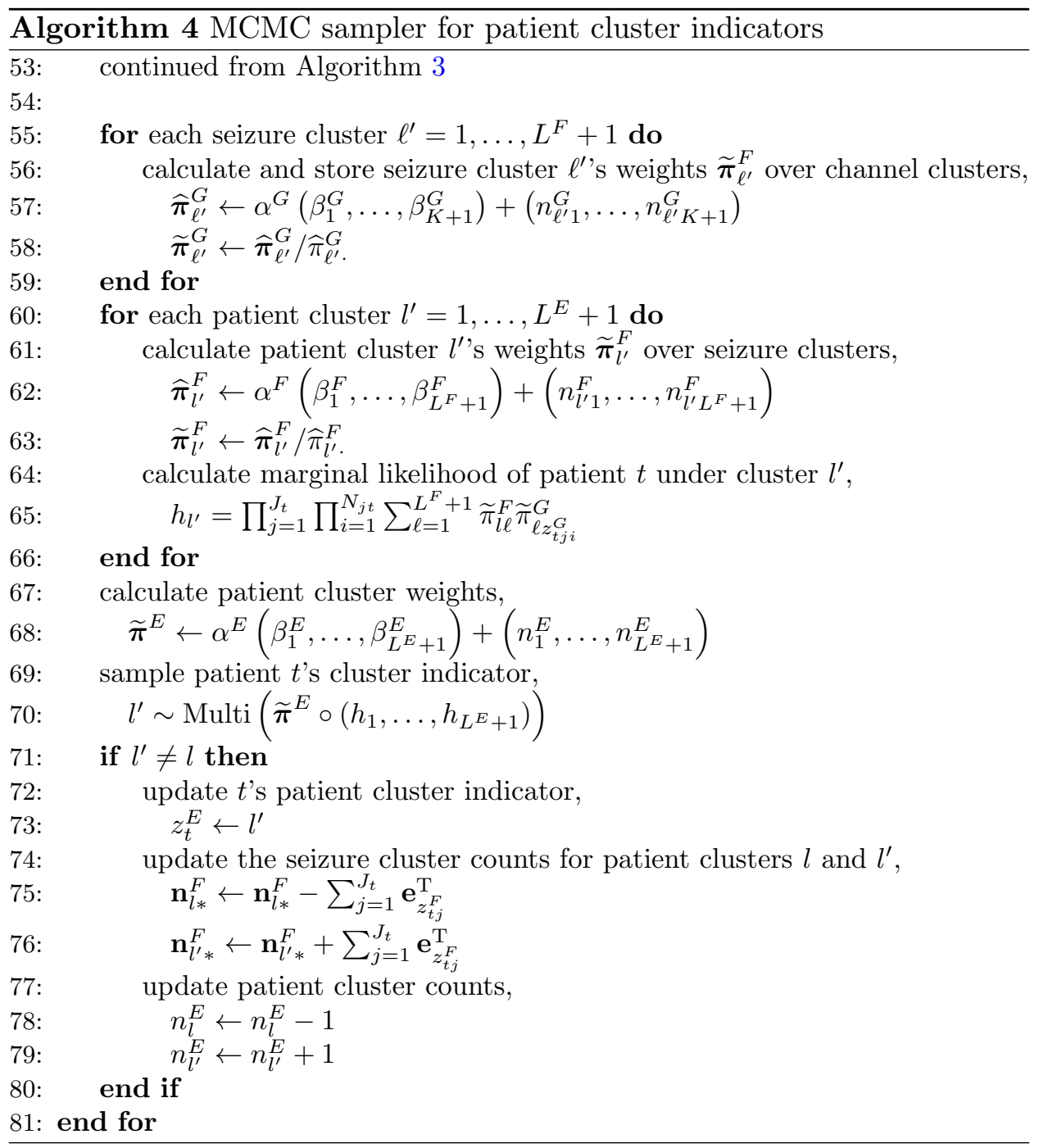




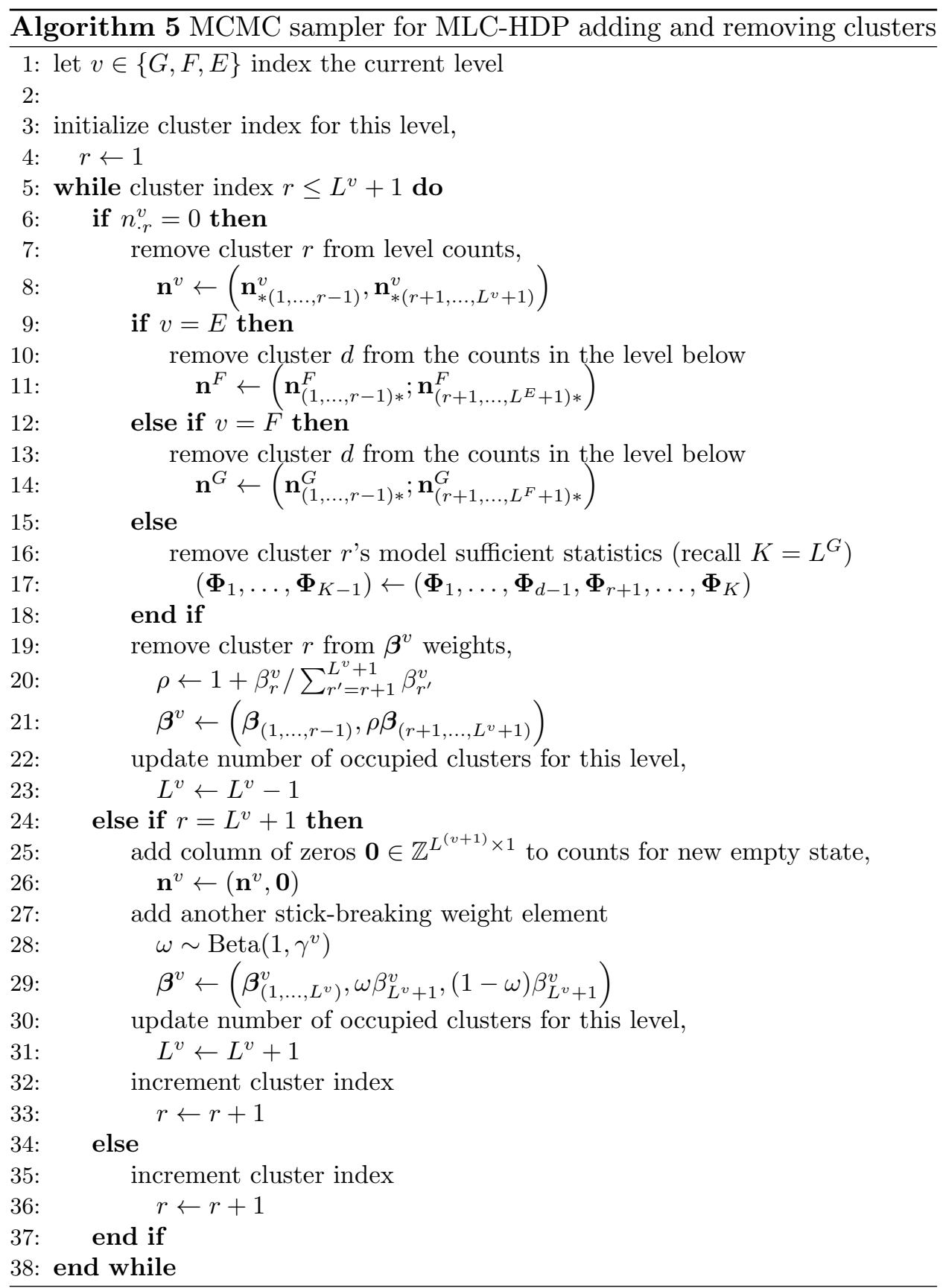



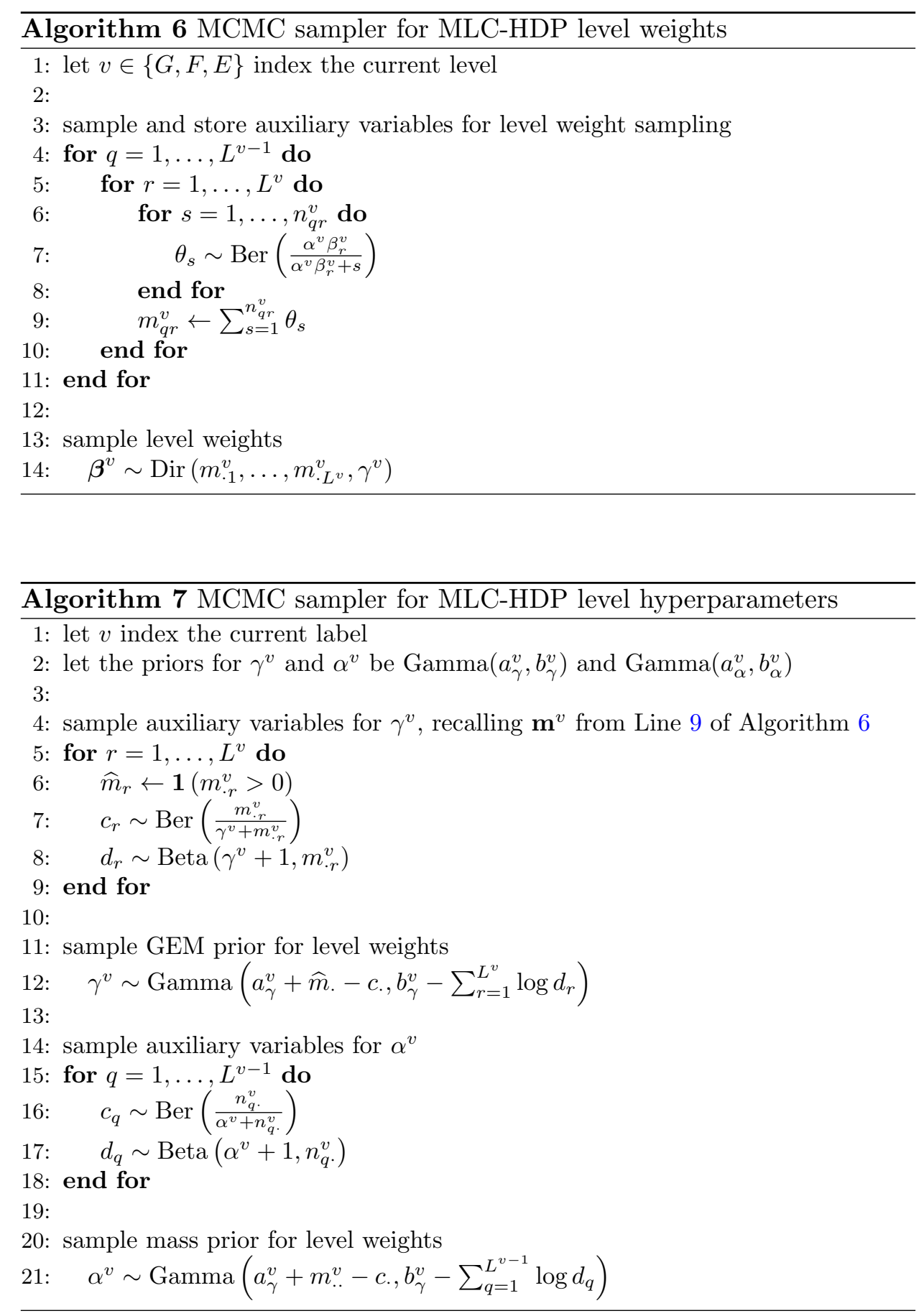


\begin{tabular}{|c|c|c|c|c|c|c|c|c|c|c|c|c|}
\hline \multirow[b]{2}{*}{ Dist } & \multicolumn{3}{|c|}{ Comp 1} & \multicolumn{3}{|c|}{ Comp 2} & \multicolumn{3}{|c|}{ Comp 3} & \multicolumn{3}{|c|}{ Comp 4} \\
\hline & $w$ & $\mu$ & $\sigma^{2}$ & $w$ & $\mu$ & $\sigma^{2}$ & $w$ & $\mu$ & $\sigma^{2}$ & $w$ & $\mu$ & $\sigma^{2}$ \\
\hline $\mathrm{T} 1$ & .75 & 0 & 1.0 & .25 & 3.0 & 2.0 & & & & & & \\
\hline $\mathrm{T} 2$ & .55 & 0 & 1.0 & .45 & 3.0 & 2.0 & & & & & & \\
\hline T3 & .40 & 0 & 1.0 & .30 & -2.0 & 2.0 & .30 & 2.0 & 2.0 & & & \\
\hline $\mathrm{T} 4$ & .39 & 0 & 1.0 & .29 & -2.0 & 2.0 & .29 & 2.0 & 2.0 & .03 & 10.0 & 1.0 \\
\hline
\end{tabular}

Parameters for the true distributions (T1-T4) used in the simulation study, where $p_{T}=\sum_{i} w_{i} \mathcal{N}\left(\mu_{i}, \sigma_{i}^{2}\right)$. Components (Comp) 1-4 describe the different sets of model parameters and theirs weights in each true distribution.

4. Simulation comparison to the NDP. As our model was inspired by the nested Dirichlet process of Rodríguez, Dunson and Gelfand (2008), we first explored some properties of both approaches on simulated data to explore the meaningful differences between the two multi-level clustering models. Note that we omit the DP and HDP models in this comparison because - as previously discussed - they ultimately cannot yield the simultaneous clusterings on the channel, seizure, and patient level that we desire. We use the same simulation setting described in Rodríguez, Dunson and Gelfand (2008): observations are generated from one of four distributions (T1-T4), each of which is a mixture of two to four normal distributions, whose parameters are given in Table 1.

Our simulated dataset contains 5 samples from each of the four distributions (T1-T4), where each sample contains 100 observations from that particular distribution. We used the same hyperparameters described in Rodríguez, Dunson and Gelfand (2008), and ran 25 chains for both the MLC-HDP and NDP models, where each chain had a 5000 iteration burn-in and thinning down to every 10th iteration, gathering 10,000 samples total.

We observed that the NDP with inference described as in Rodríguez, Dunson and Gelfand (2008) exhibits high autocorrelation compared to the MLC-HDP in a few investigated common parameters, as shown in the top left and top middle parts of Figure 3. Anecdotal results for this simulated dataset indicate that the main cause of this additional autocorrelation is that the weights over the observation level atoms are explicitly sampled only once per iteration in the NDP, whereas they are updated continuously in the Rao-Blackwell direct assignment sampling scheme of the MLC-HDP. Results on our iEEG seizure dataset presented in Section ?? support this hypothesis. Nevertheless, we hesitate to make any strong claims about the autocorrelation inherent in the NDP mode versus the MLC-HDP model since autocorrelation is so tied to the particular inference scheme (Rao-

imsart-aoas ver. 2011/12/06 file: mlchdpAOAS.supp_v05.tex date: January 3, 2016 

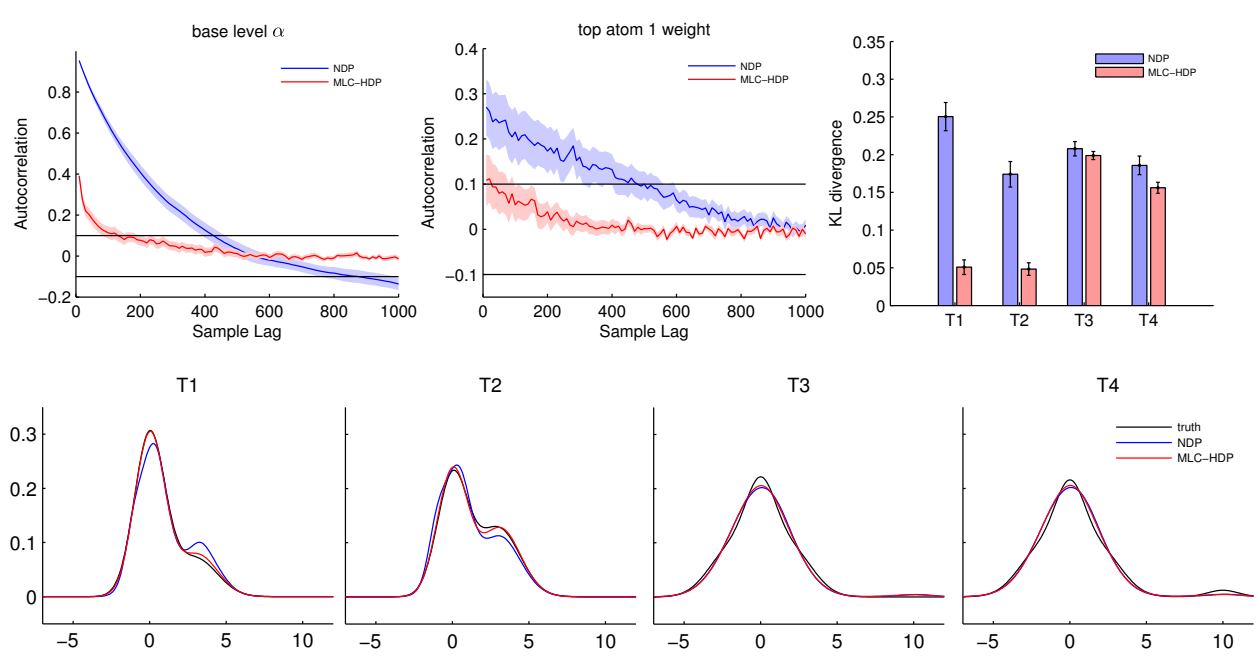

FIG 3. Autocorrelation plots for the lower level concentration parameter $\alpha$ (top left) and the weight on the first top-level mixture (top middle) for the NDP and MLC-HDP models. The dark lines of these plots are the mean over the 25 chains, the shaded region denotes the standard error. (top right:) the mean Kullback-Leibler (KL) divergence in each of the four distributions for the NDP and MLC-HDP. Error bars bound the region of one standard error. (bottom row:) true and average estimated density functions for each of the four true GMM distributions.

Blackwellized, explicitly-sampled weights, adaptive Gibbs sampling, etc) and could potentially be improved by another.

The bottom row of Figure 3 shows the MLC-HDP and NDP estimated density functions for each of the four distributions along with the true density functions. The MLC-HDP usually found three top-level groups, and the NDP split evenly between two and three top-level groups, consistent with the result Rodríguez, Dunson and Gelfand (2008) give in their Figure 3 for $J=20$ and $n=100$. With this number of observations and samples, both methods were unable to distinguish the T3 and T4 groups, which differ only in a small additional mode at $x=10$ in the fourth distribution. We use the Kullback-Leibler divergence $D_{\mathrm{KL}}$ (true $\|$ estimated) (Mackay 2003, pg. 34) for each of the four distributions between the true density functions and the estimated density functions to assess the degree of similarity. These Kullback-Leibler results, shown in the top right of Figure 3, illustrate how the estimates of the MLC-HDP model are better than those of the NDP, especially in $\mathrm{T} 1$ and $\mathrm{T} 2$.

The simulated dataset originally designed by Rodríguez, Dunson and Gelfand (2008) contains common lower level distributions between differ- 
ent top level mixtures. For example T1 and T2 contain data from the same two normal distributions, just in different proportion. The NDP must estimate those base distributions independently for $\mathrm{T} 1$ and $\mathrm{T} 2$, whereas the MLC-HDP estimates benefit from the data between all the groups.

When attempting to scale up the NDP to a modestly sized $(K=55$, $L^{F}=35$, and $L^{E}=35$ ) three-level structure for our iEEG seizure dataset, we found that the total number of observation atoms - more than $67,000-$ quickly became computationally impractical. The MLC-HDP avoids this pitfall by sharing observation level atoms among the higher level mixtures.

5. Schiff seizure features. The following six features were used by Schiff et al. (2005) to quantify the spatiotemporal dynamics of a seizure. See their paper for more details. Let $x_{i}[t]$ denote the $i$ th channel's voltage at each sample $t$ in a given time window. Let $N$ denote the number of channels in the seizure and $T$ the number of samples in a time window.

Total power is the sum of the squared values at each time point

$$
P=\sum_{i=1}^{N} \sum_{t=1}^{T} x_{i}[t]^{2} 1
$$

Total correlation at zero time lag find the zero-lag correlation across channels after first-order detrending each channel's time series. Let $a_{i} t+b_{i}$ be the first order trend for chanel $i$ and $y_{i}[t]=x_{i}[t]-a_{i} t-b_{i}$, so the normalized cross-correlation between channels $i$ and $j$ is given by

$$
c_{i, j}(\tau)=\frac{\sum_{t=-T / 2}^{T / 2} y_{i}[t] \cdot y_{j}[t+\tau]}{\left(\sum_{t=-T / 2}^{T / 2}\left(y_{i}[t]\right)^{2}\right)^{1 / 2}\left(\sum_{t=-T / 2}^{T / 2}\left(y_{j}[t]\right)^{2}\right)^{1 / 2}}
$$

The total zero-lag cross-correlation is thus

$$
S_{0}=\sum_{\{i, j=1, \ldots, N \mid i \neq j\}}\left|c_{i, j}(\tau=0)\right|
$$

Total correlation at arbitrary time lag sums the correlation values larger than twice the estimator of the standard deviation, which is given as

$$
\sigma_{i, j}=\frac{1}{T+1-\tau}\left|\sum_{\tau=-T / 2}^{T / 2} c_{i, i}[\tau] \cdot c_{j, j}[\tau]\right|^{1 / 2},
$$


and thus the total correlation at arbitrary lag is

$$
S_{\tau}=\sum_{\{i, j=1, \ldots, N \mid i \neq j\}} \sum_{\tau=-T / 2}^{T / 2} c_{i, j}[\tau] \cdot \mathbf{1}\left(c_{i, j}[\tau]>2 \sigma_{i, j}\right)
$$

Phase amplitude coherence reflects the average phase amplitude across all the channels and time points. We calculate the phase $\varphi_{i}[t]$ of channel $i$ at time $t$ using the Hilbert transform. Let

$$
r[t]=\frac{1}{N} \sqrt{\left(\sum_{i=1}^{N} \cos \left(\varphi_{i}[t]\right)\right)^{2}+\left(\sum_{i=1}^{N} \sin \left(\varphi_{i}[t]\right)\right)^{2}}
$$

denote the average phase amplitude at time $t$ over all the channels. The phase amplitude coherance is simply the average over all time points,

$$
\mathbb{E}[r]=\frac{1}{T} \sum_{t=1}^{T} r[t]
$$

Phase angle dispersion is a metric on the average phase angle across all the channels and time points. Let

$$
\theta[t]=\tan ^{-1}\left(\left(\sum_{i=1}^{N} \cos \left(\varphi_{i}[t]\right)\right)+\left(\sum_{i=1}^{N} \sin \left(\varphi_{i}[t]\right)\right)\right)
$$

denote the average phase angle at time $t$, with $\theta^{\prime}[t]$ representing the non-discontinuous, unwrapped version of the phase. The dispersion of the phase angle is given by the variance of the first-order differences,

$$
\theta_{\text {disp }}=\mathbb{V}[\{\Delta \theta[t] \mid \Delta \theta[t]=\theta[t+1]-\theta[t], t=1, \ldots, T-1\}]
$$

Phase amplitude dispersion is a similar metric for the phase amplitude,

$$
r_{\text {disp }}=\mathbb{V}[\{\Delta r[t] \mid \Delta r[t]=r[t+1]-r[t], t=1, \ldots, T-1\}]
$$

6. Alternative sampling schemes. Throughout our paper we use a collapsed Rao-Blackwellized (RB) sampler when possible, following Teh et al. (2006) and previous literature (Gelfand and Smith 1990; Liu, Wong and Kong 1994; Casella and Robert 1996). Nevertheless, we are interested in the properties of different sampling schemes for our application and so tested convergence and autocorrelation properties for four different combinations of

imsart-aoas ver. 2011/12/06 file: mlchdpAOAS.supp_v05.tex date: January 3, 2016 

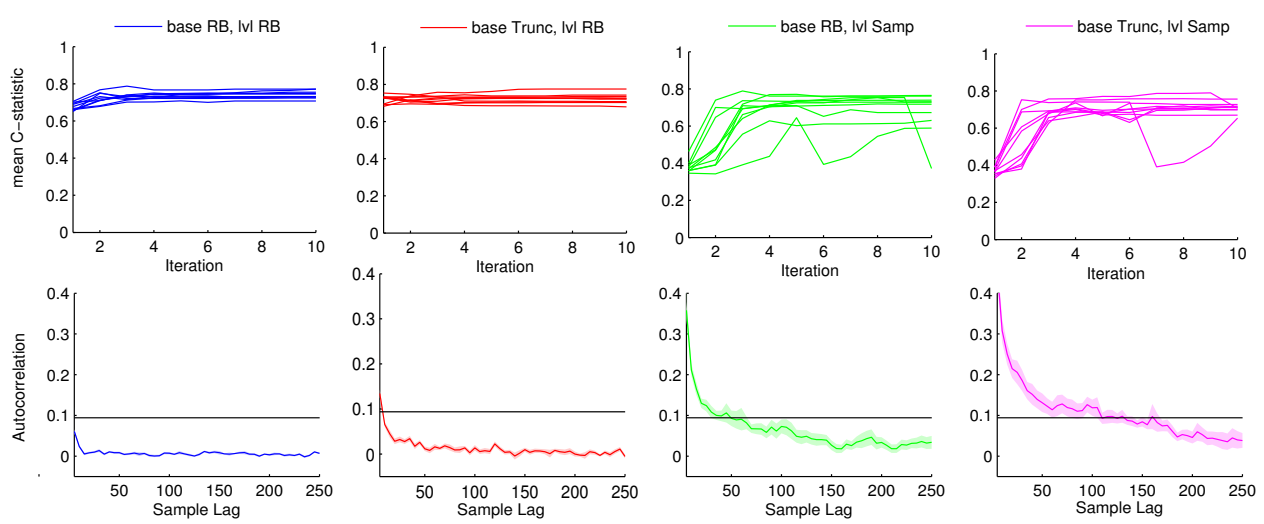

FIG 4. A comparison between collapsed Rao-Blackwelled (RB) direct assignment and explicit (Trunc or Samp) samplers for the observation atom parameters $\phi_{k}$ and the level atom weights $\boldsymbol{\pi}$. Each column represents one of the four possible combinations of base-and levelsamplers. The top row shows the convergence of average seizure clustering performance for 10 chains in the first $10 \mathrm{MCMC}$ iterations. The bottom row shows the average autocorrelation of the observation level concentration parameters $\alpha^{G}$ over 250 iteration lags, where the upper confidence bound is shown as a black line.

observation and higher-level Gibbs samplers. For the observation atoms, we compared the collapsed RB sampler to a common truncated version (Ishwaran and James 2001, 2002; Sudderth 2006), where we approximate the infinite number of atoms with a (large) finite number whose parameters we explicitly sample. This truncated sampler is used by Rodríguez, Dunson and Gelfand (2008) in their NDP model. For the higher levels, we either use the collapsed RB sampler or the explicitly sample the weight parameters from the Dirichlet posterior. For each of these four sampler combinations, we investigated the convergence of the average seizure clustering performance and the autocorrelation of the lowest concentration parameter $\alpha^{E}$.

Figure 4 shows the results from these experiments. We first note that explicitly sampling the higher-level weight parameters (shown in the third and fourth columns of Figure 4) seems to have the most pernicious effect on both convergence and autocorrelation. At the higher levels of the model, where patient and seizure counts are relatively small (10 patients and 193 seizures versus 21,476 individual channel-activity observations), the Dirichlet posterior for the level weights has relatively low counts in each of the components, and so the variability of the sampled weights is quite high. This high variability is most likely the cause of the slower convergence and higher autocorrelation results.

We next note that the differences between the two observation atom sam-

imsart-aoas ver. 2011/12/06 file: mlchdpAOAS.supp_v05.tex date: January 3, 2016 
plers is much smaller than those of the two level weight samplers. Interestingly, the observation-level truncated sampler seems to have slightly better convergence properties than the RB sampler. This result is similar to that of an analogous experiment in Fox et al. (2011). In that paper, the authors argue that sampling the cluster indicators all at once instead of one at a time (as in the RB sampler) improves mixing and thus convergence. In our seizure application, this also seems to be the case, though the differences are small. Our results also indicate that slight improvement in convergence of the truncated sampler comes at the cost of slightly increased autocorrelation. Anecdotally, we have found that in smaller (simulated) datasets, the higher autocorrelation is more pronounced.

7. The influence of individual patients. One of the motivating ideas behind using a hierarchical model for a collection of patients instead of a flat model is that its ability to distinguish between different groups and sub-groups within the data allows it to better balance patients with very different numbers of seizures. Alternatively, in a flat model, no distinctions among sub-groups are made, so all the seizures of all the patients are modeled together. Patients that happen to have many seizures may thus influence the model more than appropriate, and those with few seizures may influence the model less than appropriate.

To test the extent to which this skewing (or lack thereof) occurs in the MLC-HDP model, we examined the perplexity of all of patient B's seizures under a set of models, each of which had another patient removed from the training dataset. Ideally, every patient should improve the model relative to patient B's seizures, and thus when each patient is removed, the model should worsen (and the perplexity increase). We ran 25 chains, taking 200 samples from each at 20-iteration thinning after 500 burn-in iterations.

The left side of Figure 5 shows the results of this experiment for both the hierarchical MLC-HDP and flat DP models outlined in our paper. In addition to underscoring that the MLC-HDP simply yields superior models to the DP (since the perplexities are lower), we also note that the MLCHDP's range of variation from the case where none of the patients were held out is smaller. We interpret this smaller variation as a sign that the MLC-HDP model is influenced less, and also more regularly, by removing one patient from the dataset. Furthermore, the patients with relatively few numbers of seizures (A, C, and G) have an influence more balanced with the patients with relatively many seizures (D, E, and F) in the MLC-HDP. Finally, we note that the detrimental effect of patient H's seizures on the model is much smaller (and almost negligible) in the MLC-HDP than the DP 

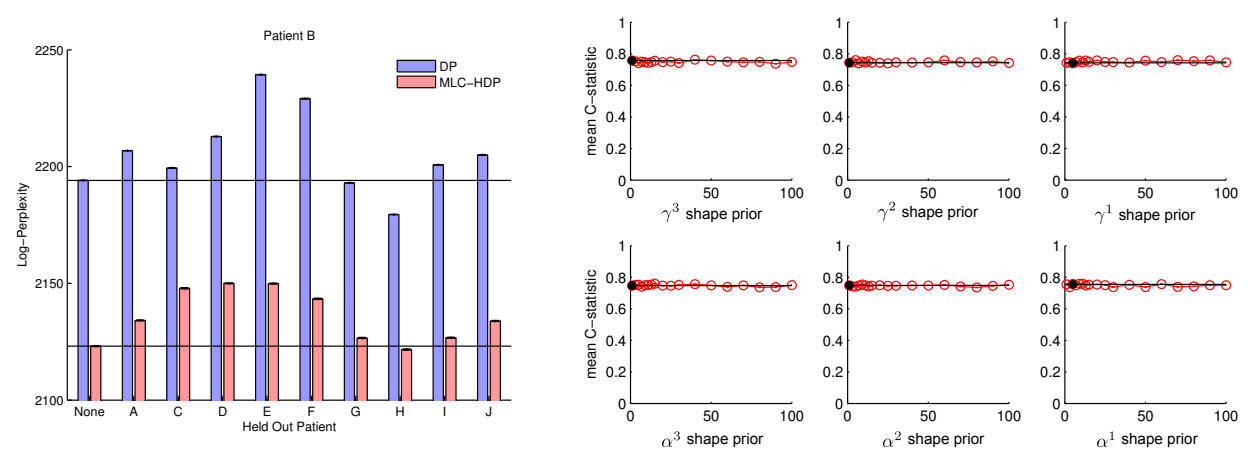

FIG 5. (left) The log-perplexity of held-out seizures from patient $B$ when also removing each of the other patients (or none) from the training set for the MLC-HDP and DP models. The horizontal black line denotes the baseline perplexity with all the other patients in the training set. Lower perplexities indicate better models. Errorbars (barely visible) denote standard error. (right) The average seizure clustering performance (C-statistic) of the MLC-HDP model when the concentration parameter Gamma prior's shape parameter for one of the $\alpha$ or $\gamma$ concentration parameters at one of three levels is varied over values between 1 and 100. The standard shape value used in the rest of this work is shown as a black dot, and its associated clustering performance is shown with a black line.

model. Recall that patient $\mathrm{H}$ was also the patient where the two physician experts disagreed about seizure clustering more than the MLC-HDP and the DP models did with either. These seizures of patient $\mathrm{H}$ do have a different form than those of most other patients, so it is not entirely surprising that they have led to outlying results in some of our experiments. 
8. Patient Seizure Images. Below are images representing the Beta band $(13-30 \mathrm{~Hz}) \log _{10}$ power over the channels (rows) at each time point (columns) of the seizures, from -30 seconds before to +90 seconds after each seizure. Seizures increase temporally from left to right and then down the rows. For those patients who had more than 20 seizures (D, E, G, H), only 20 are shown.

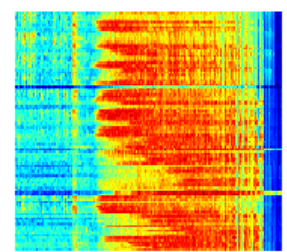

FIG 6. Patient A's only seizure.
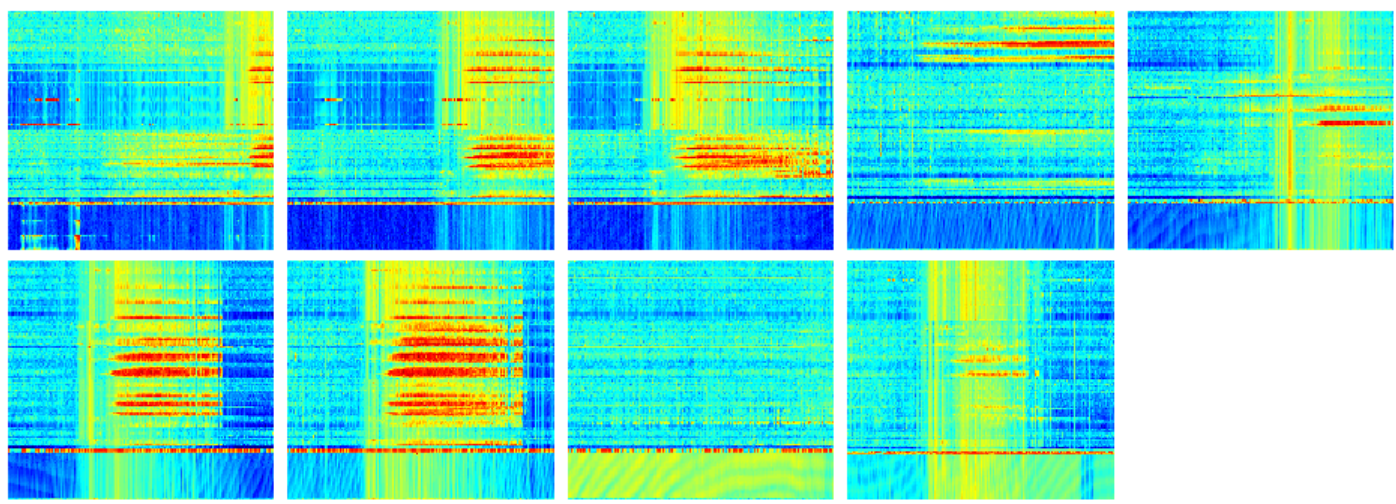

Fig 7. Patient B's 9 seizures.
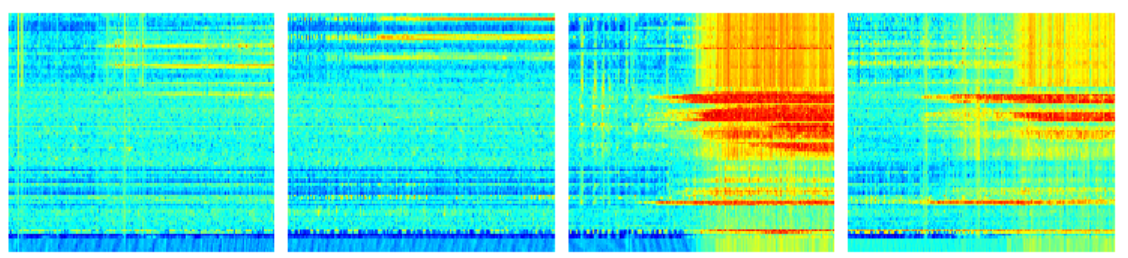

Fig 8. Patient C's 4 seizures.

imsart-aoas ver. 2011/12/06 file: mlchdpAOAS.supp_v05.tex date: January 3, 2016 

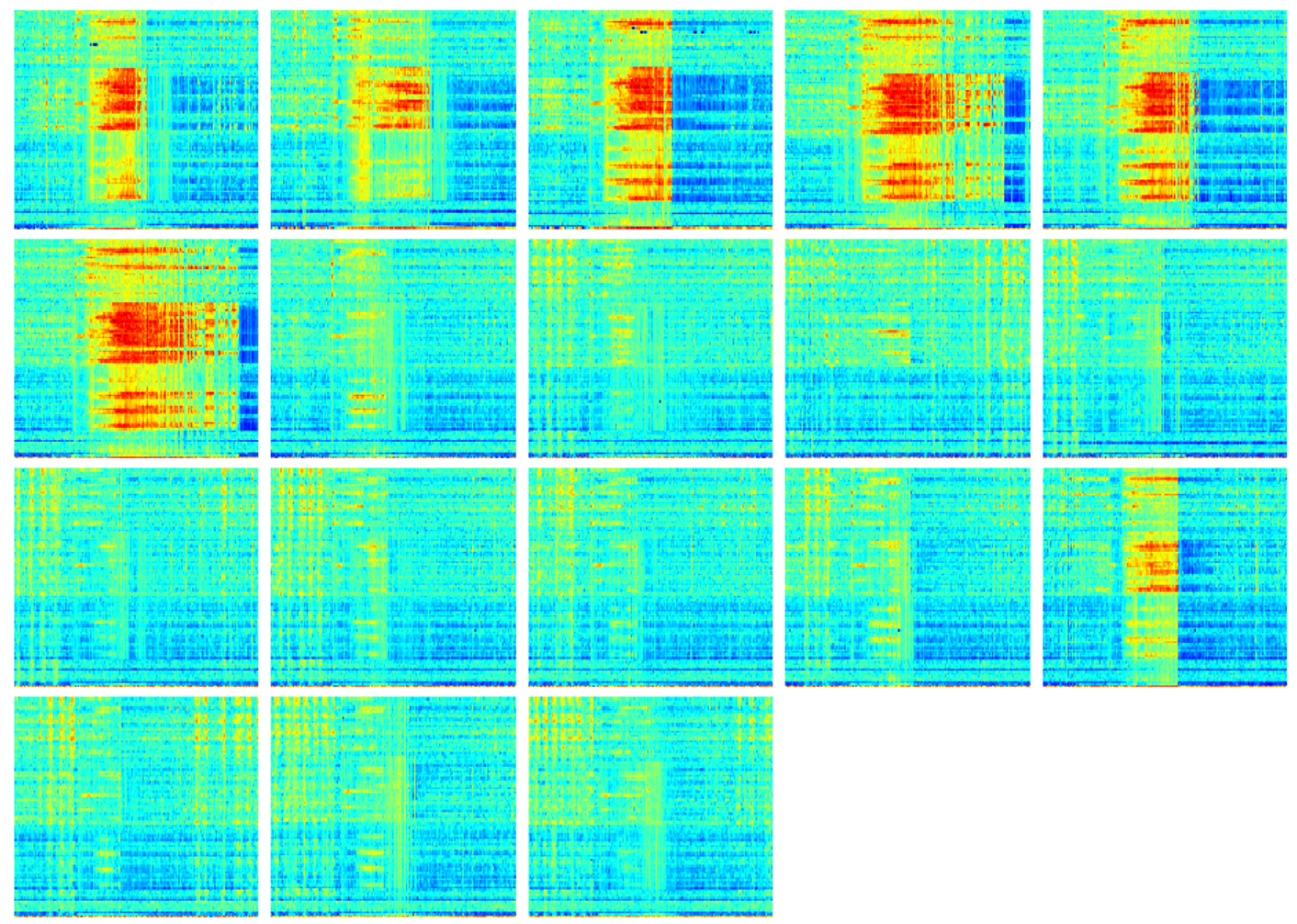

FIG 9. Patient D's 18 seizures.

\section{References.}

Casella, G. and Robert, C. P. (1996). Rao-Blackwellisation of sampling schemes. Biometrika 83 81-94.

Fox, E. B., Sudderth, E. B., Jordan, M. I. and Willsky, A. S. (2011). A sticky HDP-HMM with application to speaker diarization. The Annals of Applied Statistics $\mathbf{5}$ 1020-1056.

Gelfand, A. E. and Smith, A. F. M. (1990). Sampling-based approaches to calculating marginal densities. Journal of the American statistical association 85 398-409.

Gelman, A., Carlin, J. B., Stern, H. S. and Rubin, D. S. (2004). Bayesian Data Analysis, Second ed. Chapman \& Hall/CRC, Boca Raton, FL.

ISHWARAN, H. and JAMES, L. F. (2001). Gibbs sampling methods for stick-breaking priors. Journal of the American Statistical Association 96 161-173.

Ishwaran, H. and James, L. F. (2002). Approximate Dirichlet Process Computing in Finite Normal Mixtures. Journal of Computational and Graphical Statistics 11 508532.

LiU, J. S., Wong, W. H. and Kong, A. (1994). Covariance structure of the Gibbs sampler with applications to the comparisons of estimators and augmentation schemes. Biometrika 81 27-40.

MACKAY, D. J. C. (2003). Information Theory, Inference, and Learning Algorithms. Cam-

imsart-aoas ver. 2011/12/06 file: mlchdpAOAS.supp_v05.tex date: January 3, 2016 

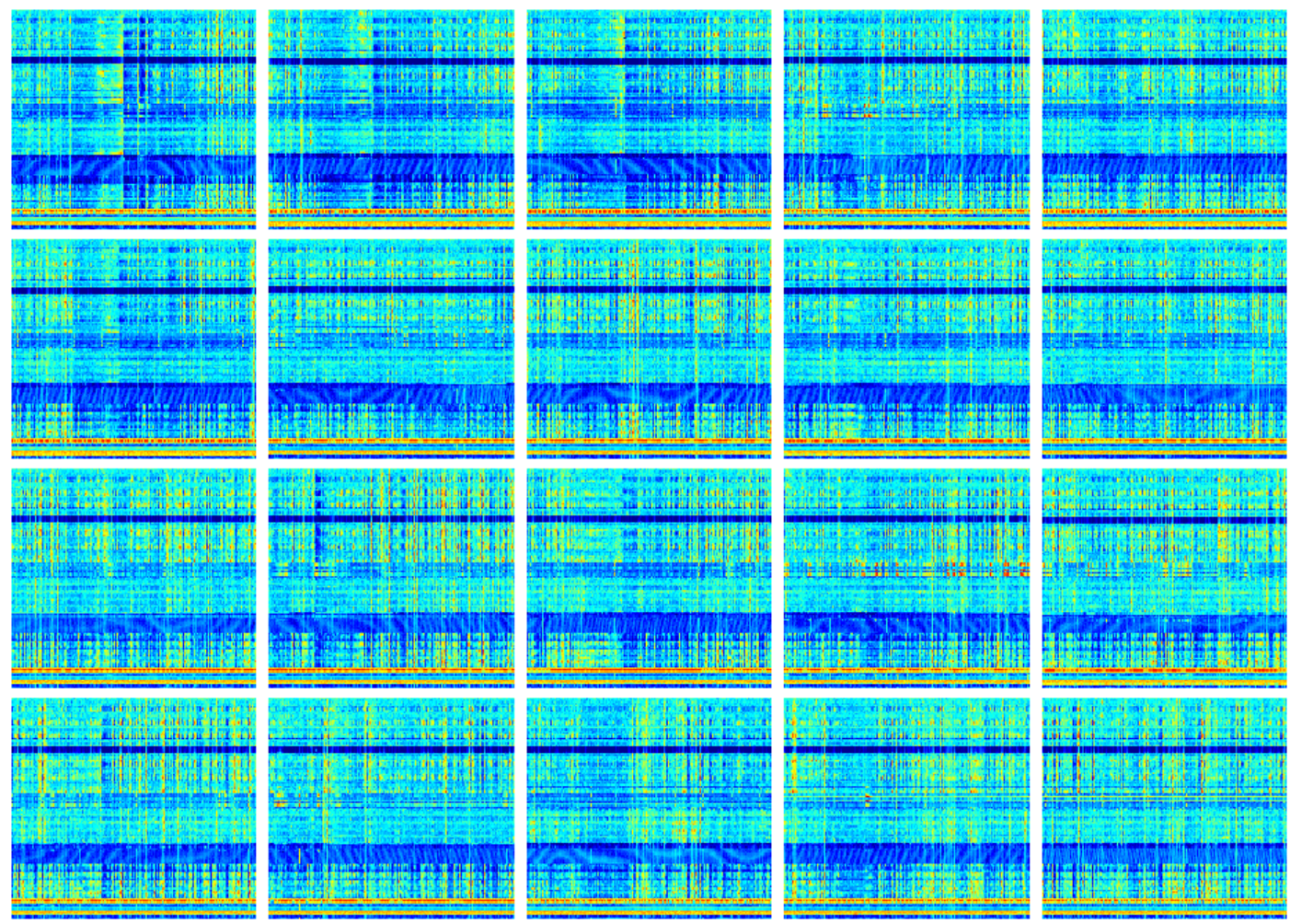

FIG 10. Patient E's first 20 of 61 seizures.

bridge University Press, Cambridge, UK.

Rodríguez, A., Dunson, D. B. and Gelfand, A. E. (2008). The Nested Dirichlet Process. Journal of the American Statistical Association 103 1131-1154.

Schiff, S. J., Sauer, T., Kumar, R. and Weinstein, S. L. (2005). Neuronal spatiotemporal pattern discrimination: the dynamical evolution of seizures. NeuroImage 28 1043-1055.

SudDerth, E. B. (2006). Graphical models for visual object recognition and tracking $\mathrm{PhD}$ thesis.

Teh, Y. W., Jordan, M. I., Beal, M. J. and Blei, D. M. (2006). Hierarchical Dirichlet processes. Journal of the American Statistical Association 101 1566-1581. 

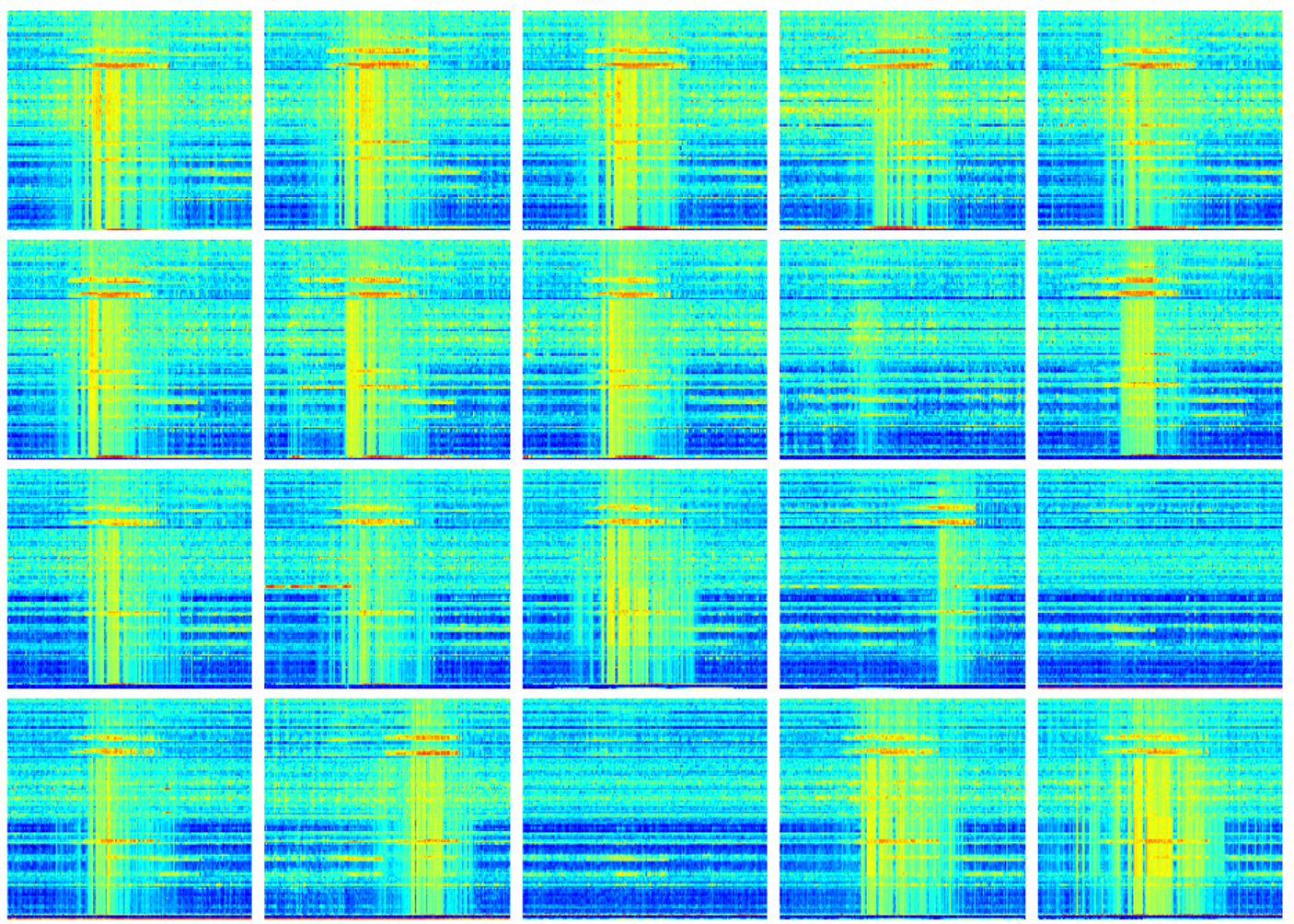

FIG 11. Patient F's first 20 of 50 seizures.

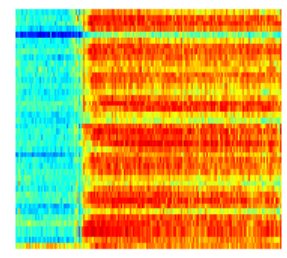

FIG 12. Patient G's only seizure.

imsart-aoas ver. 2011/12/06 file: mlchdpAOAS.supp_v05.tex date: January 3, 2016 
D. WULSIN ET AL.
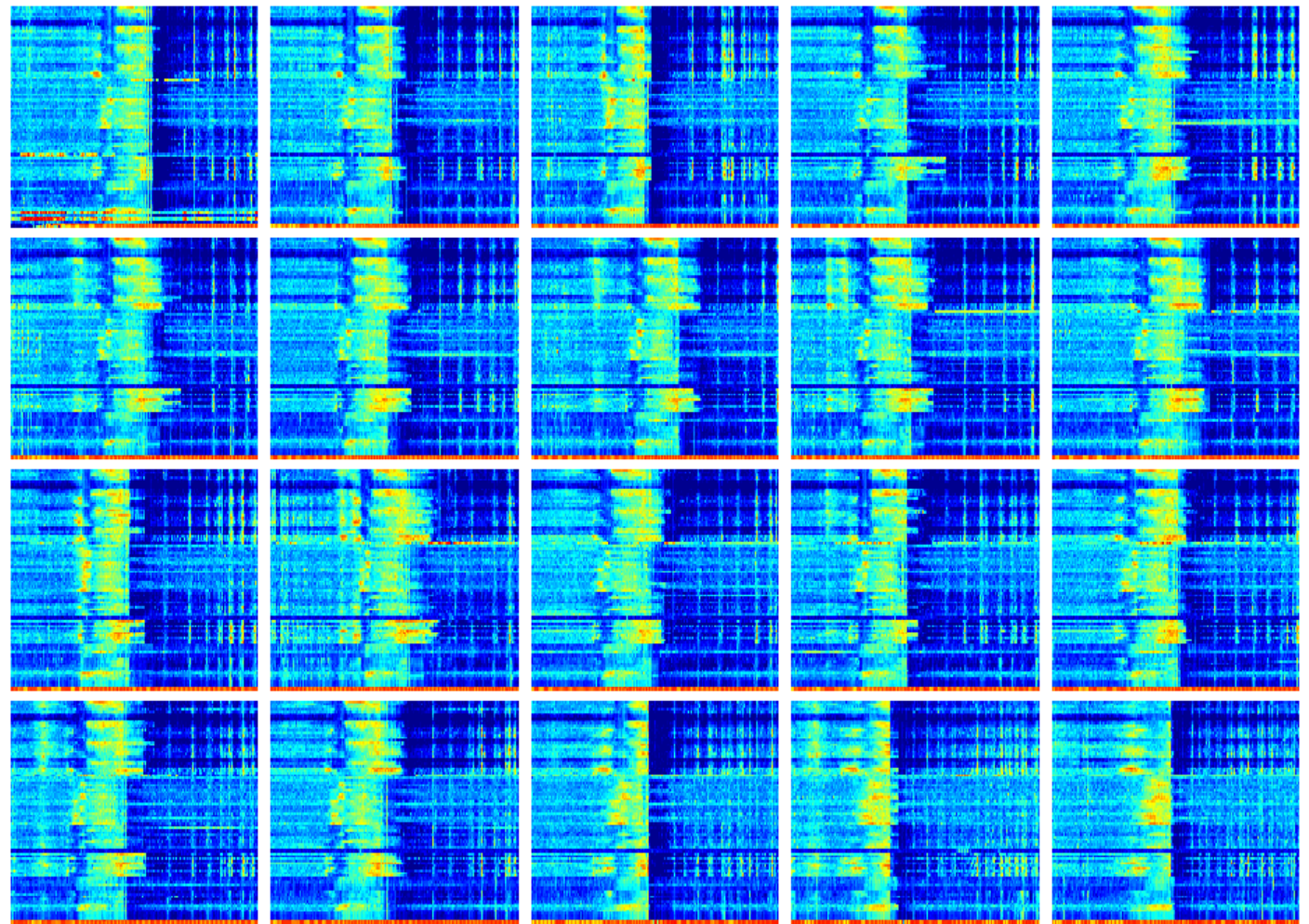

FIG 13. Patient H's fist 20 of 22 seizures.

imsart-aoas ver. 2011/12/06 file: mlchdpAOAS.supp_v05.tex date: January 3, 2016 

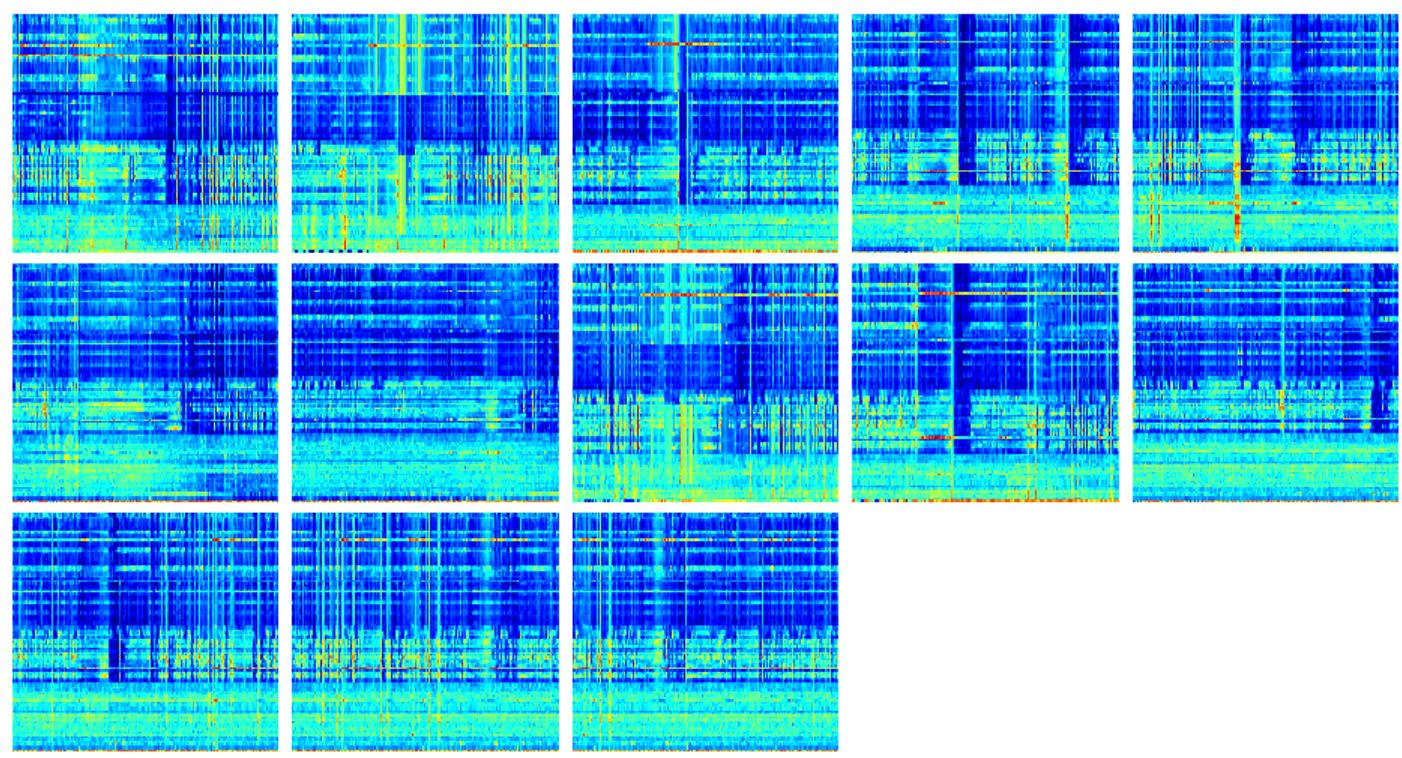

FIG 14. Patient I's 13 seizures.
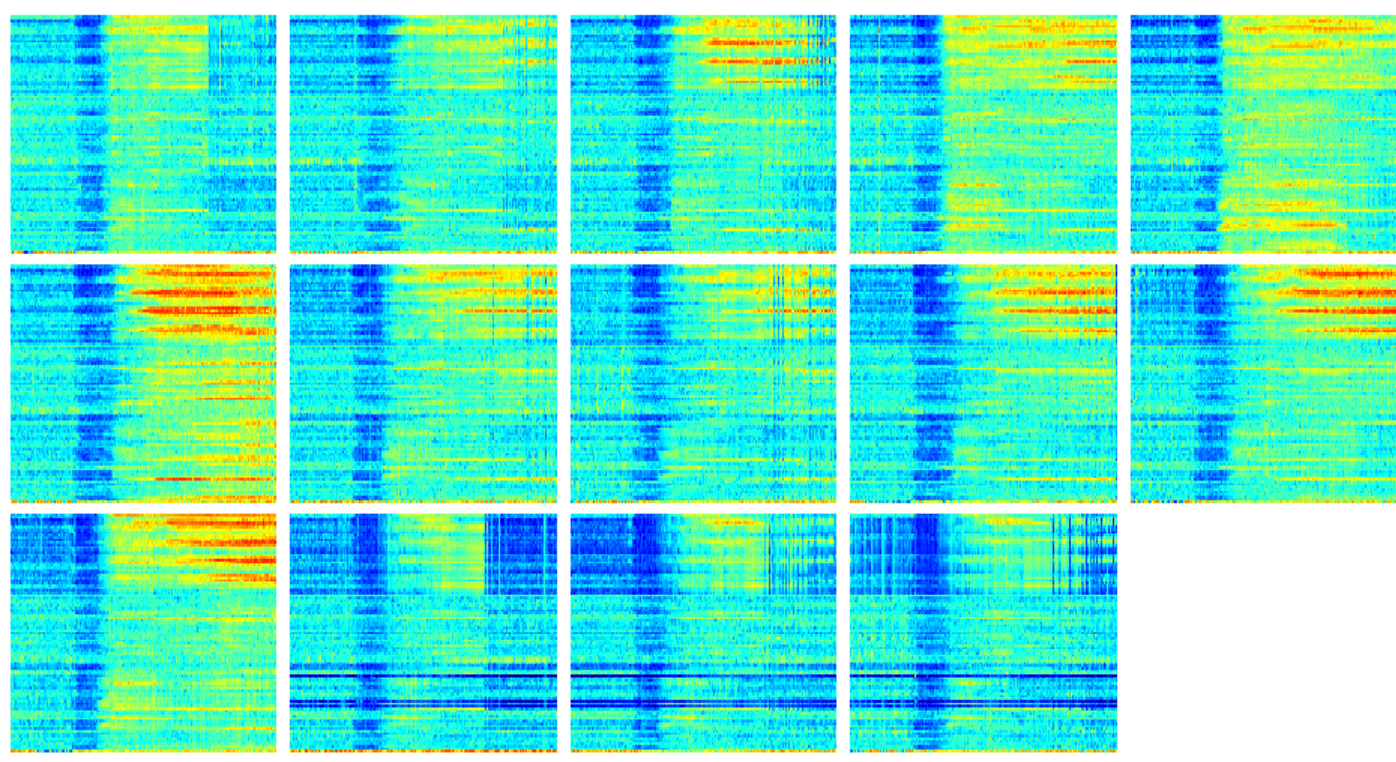

Fig 15. Patient J's 14 seizures.

imsart-aoas ver. 2011/12/06 file: mlchdpAOAS.supp_v05.tex date: January 3, 2016 